\title{
Coincidence point theorems for graph-preserving multi-valued mappings
}

Jukrapong Tiammee and Suthep Suantai*

"Correspondence:

suthep.s@cmu.ac.th

Department of Mathematics,

Faculty of Science, Chiang Mai

University, Chiang Mai, Thailand

\begin{abstract}
In this paper, we introduce the concepts of graph-preserving multi-valued mapping and a new type of multi-valued weak G-contraction on a metric space endowed with a directed graph $G$. We prove some coincidence point theorems for this type of multi-valued mapping and a surjective mapping $g: X \rightarrow X$ under some conditions. Several examples for these new concepts and some examples satisfying all conditions of our main results are also given. Our main results extend and generalize many coincidence point and fixed point theorems in partially ordered metric spaces.
\end{abstract} MSC: $47 \mathrm{H} 04 ; 47 \mathrm{H} 10$

Keywords: fixed point theorems; multi-valued mappings; partially ordered set; monotone mappings; graph-preserving

\section{Introduction}

Fixed point theory of multi-valued mappings plays an important role in science and applied science. It has applications in control theory, convex optimization, differential inclusions and economics.

For a metric space $(X, d)$, we let $C B(X)$ and $\operatorname{Comp}(X)$ be the set of all nonempty closed bounded subsets of $X$ and the set of all nonempty compact subsets of $X$, respectively. A point $x \in X$ is a fixed point a multi-valued mapping $T: X \rightarrow 2^{X}$ if $x \in T x$. The first wellknown theorem for multi-valued contraction mappings was given by Nadler in 1969 [1].

Theorem 1.1 Let $(X, d)$ be a complete metric space and let $T$ be a mapping from $X$ into $C B(X)$. Assume that there exists $k \in[0,1)$ such that

$$
H(T x, T y) \leq k d(x, y) \quad \text { for all } x, y \in X
$$

Then there exists $z \in X$ such that $z \in T z$.

Nadler's fixed point theorem for multi-valued contractive mappings has been extended in many directions (see [2-6]). Reich [7] proved the following fixed point theorem for multi-valued $\varphi$ contraction mappings.

Theorem 1.2 Let $(X, d)$ be a complete metric space and let $T$ be a mapping from $X$ into $\operatorname{Comp}(X)$. Assume that there exists a function $\varphi:[0, \infty) \rightarrow[0,1)$ such that

$$
\lim _{r \rightarrow t^{+}} \sup \varphi(r)<1 \text { for each } t \in(0, \infty)
$$

O2014Tiammee and Suantai; licensee Springer. This is an Open Access article distributed under the terms of the Creative Commons Attribution License (http://creativecommons.org/licenses/by/2.0), which permits unrestricted use, distribution, and reproduction in any medium, provided the original work is properly cited. 
and

$$
H(T x, T y) \leq \varphi(d(x, y)) d(x, y) \quad \text { for all } x, y \in X
$$

Then there exists $z \in X$ such that $z \in T z$.

The multi-valued mapping $T$ considered by Reich [7] in Theorem 1.2 has compact values, that is, $T x$ is a nonempty compact subset of $X$ for all $x \in X$. In 1988, Mizoguchi and Takahashi [8] relaxed the compactness assumption on $T$ to closed and bounded subsets of $X$. They proved the following theorem which is a generalization of Nadler's theorem.

Theorem 1.3 Let $(X, d)$ be a complete metric space and let $T: X \rightarrow C B(X)$. Assume that there exists a function $\varphi:[0, \infty) \rightarrow[0,1)$ such that

$$
\lim _{r \rightarrow t^{+}} \sup \varphi(r)<1 \quad \text { for each } t \in[0, \infty)
$$

and

$$
H(T x, T y) \leq \varphi(d(x, y)) d(x, y) \quad \text { for all } x, y \in X
$$

Then there exists $z \in X$ such that $z \in T z$.

In 2007, Berinde and Berinde [3] extended Theorem 1.1 to the class of multi-valued weak contractions.

Definition 1.4 ([3]) Let $(X, d)$ be a metric space and $T: X \rightarrow C B(X)$ be a multi-valued mapping. $T$ is said to be a multi-valued weak contraction or a multi-valued $(\theta, L)$-weak contraction if there exist two constants $\theta \in(0,1)$ and $L \geq 0$ such that

$$
H(T x, T y) \leq \theta d(x, y)+L d(y, T x) \quad \text { for all } x, y \in X
$$

They proved in [3], Theorem 3 that in a complete metric space every multi-valued $(\theta, L)$ weak contraction has a fixed point. In the same paper, they also introduced a class of multivalued mappings which is more general than that of weak contractions.

Definition 1.5 ([3]) Let $(X, d)$ be a metric space and $T: X \rightarrow C B(X)$ a multi-valued mapping. $T$ is said to be a generalized multi-valued $(\alpha, L)$-weak contraction if there exist $L \geq 0$ and a function $\alpha:[0, \infty) \rightarrow[0,1)$ satisfying $\lim \sup _{r \rightarrow t^{+}} \alpha(r)<1$, for every $t \in[0, \infty)$, such that

$$
H(T x, T y) \leq \alpha(d(x, y)) d(x, y)+L d(y, T x) \quad \text { for all } x, y \in X
$$

They also showed that in a complete metric space, every generalized multi-valued $(\alpha, L)$ weak contraction has a fixed point (see [3, Theorem 4]).

For the last ten years, many results concerning the existence of fixed points of both single-valued and multi-valued mappings in metric spaces endowed with a partial ordering have been established. The first result in this direction was given by Ran and Reurings 
[9] and they also presented its applications to linear and nonlinear matrix equations. After that many authors extended those results and studied fixed point theorems in partially ordered metric spaces (see [9-13]).

In 2008, Jachymski [14] introduced the concept of $G$-contraction and proved some fixed point results of $G$-contractions in a complete metric space endowed with a graph.

Definition 1.6 ([14]) Let $(X, d)$ be a metric space and let $G=(V(G), E(G))$ be a directed graph such that $V(G)=X$ and $E(G)$ contains all loops, i.e., $\triangle=\{(x, x): x \in X\} \subseteq E(G)$.

We say that a mapping $f: X \rightarrow X$ is a $G$-contraction if $f$ preserves edges of $G$, i.e.,

$$
x, y \in X, \quad(x, y) \in E(G) \quad \Rightarrow \quad(f(x), f(y)) \in E(G)
$$

and there exists $\alpha \in(0,1)$ such that

$$
x, y \in X, \quad(x, y) \in E(G) \quad \Rightarrow \quad d(f(x), f(y)) \leq \alpha d(x, y) .
$$

He showed in [14] that under some certain properties on $(X, d, G)$, a $G$-contraction $f: X \rightarrow$ $X$ has a fixed point if and only if $X_{f}:=\{x \in X:(x, f(x)) \in E(G)\}$ is nonempty. The mapping $f: X \rightarrow X$ satisfying condition (1.1) is also called a graph-preserving mapping.

Recently, Beg and Butt [5] introduced the concept of $G$-contraction for a multi-valued mapping $T: X \rightarrow C B(X)$ and proved some fixed point results of this kind of mappings.

Definition 1.7 ([5]) Let $T: X \rightarrow C B(X)$ be a multi-valued mapping. The mapping $T$ is said to be a $G$-contraction if there exists $k \in(0,1)$ such that

$$
H(T x, T y) \leq k d(x, y) \quad \text { for all }(x, y) \in E(G)
$$

and if $u \in T x$ and $v \in T y$ are such that

$$
d(u, v) \leq k d(x, y)+\alpha \quad \text { for each } \alpha>0,
$$

then $(u, v) \in E(G)$.

They also showed that if $(X, d)$ is a complete metric space and a triple $(X, d, G)$ has Property A [14], then a $G$-contraction mapping $T: X \rightarrow C B(X)$ has a fixed point if and only if $X_{F}:=\{x \in X:(x, y) \in E(G)$ for some $y \in T x\}$ is nonempty.

Recently, in 2013, Dinevari and Frigon [6] introduced a new concept of G-contraction which is weaker than that of Beg and Butt [5].

Definition 1.8 ([6]) Let $T: X \rightarrow 2^{X}$ be a map with nonempty values. We say that $T$ is a $G$-contraction (in the sense of Dinevari and Frigon) if there exists $\alpha \in(0,1)$ such that

$\left(C_{G}\right)$ for all $(x, y) \in E(G)$ and all $u \in T x$, there exists $v \in T y$ such that

$$
(u, v) \in E(G) \quad \text { and } \quad d(u, v) \leq \alpha d(x, y) .
$$


They showed that under some properties on a metric space which is weaker than Property A, a multi-valued G-contraction with closed values has a fixed point (see [6], Theorem 2.10 and Corollary 2.11). We note that the concept of G-contraction for multi-valued mappings does not concern the concept of graph-preserving as seen for single-valued mappings. Motivated by this observation and those previous works, we are interested in introducing the concept of graph-preserving for multi-valued mappings and study their fixed point theorem in a complete metric space endowed with a graph.

\section{Preliminaries}

Let $(X, d)$ be a metric space and $C B(X)$ be the set of all nonempty closed bounded subsets of $X$. For $x \in X$ and $A, B \in C B(X)$, define

$$
\begin{aligned}
& d(x, A)=\inf \{d(x, y): y \in A\}, \\
& \delta(A, B)=\sup \{d(x, B): x \in A\} .
\end{aligned}
$$

Denote by $H$ the Pompeiu-Hausdorff metric induced by $d$, see [4], that is,

$$
H(A, B)=\max \left\{\sup _{u \in A} d(u, B), \sup _{v \in B} d(v, A)\right\} .
$$

The following two lemmas, which can be found in [1] or [8], are useful for our main results.

Lemma 2.1 ([1]) Let $(X, d)$ be a metric space. If $A, B \in C B(X)$ and $a \in A$, then, for each $\epsilon>0$, there exists $b \in B$ such that

$$
d(a, b) \leq H(A, B)+\epsilon .
$$

Lemma 2.2 ([8]) Let $(X, d)$ be a metric space in $C B(X),\left\{x_{k}\right\}$ be a sequence in $X$ such that $x_{k} \in A_{k-1}$. Let $\alpha:[0, \infty) \rightarrow[0,1)$ be a function satisfying $\limsup _{r \rightarrow t^{+}} \alpha(r)<1$ for every $t \in$ $[0, \infty)$. Suppose that $d\left(x_{k-1}, x_{k}\right)$ is a non-increasing sequence such that

$$
\begin{aligned}
& H\left(A_{k-1}, A_{k}\right) \leq \alpha\left(d\left(x_{k-1}, x_{k}\right)\right) d\left(x_{k-1}, x_{k}\right), \\
& d\left(x_{k}, x_{k+1}\right) \leq H\left(A_{k-1}, A_{k}\right)+\alpha^{n_{k}}\left(d\left(x_{k-1}, x_{k}\right)\right),
\end{aligned}
$$

where $n_{1}<n_{2}<\cdots$ and $k, n_{k} \in \mathbb{N}$. Then $\left\{x_{k}\right\}$ is a Cauchy sequence in $X$.

Let $G=(V(G), E(G))$ be a directed graph where $V(G)$ is a set of vertices of the graph and $E(G)$ be a set of its edges. Assume that $G$ has no parallel edges. If $x$ and $y$ are vertices in $G$, then a path in $G$ from $x$ to $y$ of length $n \in \mathbb{N} \cup\{0\}$ is a sequence $\left\{x_{i}\right\}_{i=0}^{n}$ of $n+1$ vertices such that $x_{0}=x, x_{n}=y,\left(x_{i-1}, x_{i}\right) \in E(G)$ for $i=1,2, \ldots, n$. A graph $G$ is connected if there is a path between any two vertices of $G$.

A partial order is a binary relation $\leq$ over the set $X$ which satisfies the followings conditions:

1. $x \leq x$ (reflexivity);

2. If $x \leq y$ and $y \leq x$, then $x=y$ (antisymmetry);

3. If $x \leq y$ and $y \leq z$, then $x \leq z$ (transitivity) 
for all $x, y \in X$. A set with a partial order $\leq$ is called a partially ordered set. We write $x<y$ if $x \leq y$ and $x \neq y$.

Definition 2.3 Let $(X, \leq)$ be a partially ordered set. For each $A, B \subset X$,

$A \prec B$ if $a<b$ for any $a \in A, b \in B$.

Definition 2.4 Let $(X, d)$ be a metric space endowed with a partial order $\leq$. Let $g: X \rightarrow X$ be surjective and $T: X \rightarrow C B(X), T$ is said to be $g$-increasing if for any $x, y \in X$,

$$
g(x)<g(y) \quad \Rightarrow \quad T x \prec T y .
$$

In the case $g=I_{X}$, the identity map, the mapping $T$ is called an increasing mapping.

Example 2.5 Let $X=\mathbb{N}$ have the usual relation $\leq$ and $T: \mathbb{N} \rightarrow 2^{\mathbb{N}}$ and $g: X \rightarrow X$ be defined by

$$
T x=\{x+1, x+2\}, \quad x \in X,
$$

$g(1)=1$ and $g(x)=x-1$ for $x \neq 1$. It is easy to see that $T$ is $g$-increasing.

Definition 2.6 Let $X$ be a nonempty set and $G=(V(G), E(G))$ be a graph such that $V(G)=X$, and let $T: X \rightarrow C B(X)$. Then $T$ is said to be graph-preserving if

$$
(x, y) \in E(G) \quad \Rightarrow \quad(u, v) \in E(G) \quad \text { for all } u \in T x \text { and } v \in T y \text {. }
$$

Example 2.7 Let $G=(\mathbb{N}, E(G))$, where $E(G)=\{(2 n-1,2 n+1): n \in \mathbb{N}\} \cup\{(2 n, 2 n+2): n \in$ $\mathbb{N}\} \cup\{(2 n, 2 n+4): n \in \mathbb{N}\} \cup\{(2 n, 2 n): n \in \mathbb{N}\} \cup\{(1,1),(4,2)\}$. Define $T: \mathbb{N} \rightarrow C B(\mathbb{N})$ by

$$
T(n)= \begin{cases}\{2 k, 2 k+2\} & \text { if } n=2 k-1, k \in \mathbb{N} \\ \{1\} & \text { if } n=2 k, k \in \mathbb{N} .\end{cases}
$$

We will show that $T$ is a graph-preserving mapping. Let $(x, y) \in E(G)$.

If $(x, y)=(2 k, 2 k+2)$ or $(x, y)=(2 k, 2 k+4)$ or $(x, y)=(2 k, 2 k)$ or $(4,2)$, where $k \in \mathbb{N}$, then $T x=T y=\{1\}$ and $(1,1) \in E(G)$.

If $(x, y)=(2 k-1,2 k+1), k \in \mathbb{N}$, then $T x=\{2 k, 2 k+2\}, T y=\{2 k+2,2 k+4\}$ and $(2 k$, $2 k+2) \in E(G),(2 k, 2 k+4) \in E(G),(2 k+2,2 k+2) \in E(G),(2 k+2,2 k+4) \in E(G)$. And we see that $(1,1) \in E(G), T 1=\{2,4\}$ and $(2,2),(2,4),(4,2),(4,4) \in E(G)$. Hence $T$ is graphpreserving.

Example 2.8 Let $G=(X, E(G))$, where $X=\{1,2,3,4,6,8\}$ and $E(G)=\{(1,1),(1,3)\} \cup$ $\{(2,2),(2,4),(2,6),(2,8),(4,2),(4,4),(4,8),(6,8)\}$. Define $T: X \rightarrow C B(X)$ by

$$
T(x)= \begin{cases}\{2,4\} & \text { if } x=1,4 \\ \{6,8\} & \text { if } x=3 \\ \{2\} & \text { if } x=2,6,8\end{cases}
$$


It is easy to see that $T$ is graph-preserving but not $G$-contraction in the sense of Dinevari and Frigon [6] since $d(u, v)>\alpha d(1,3)$ for all $u \in T 1=\{2,4\}$ and $v \in T 3=\{6,8\}$ for any $\alpha \in(0,1)$.

Definition 2.9 Let $X$ be a nonempty set and $G=(V(G), E(G))$ be a graph such that $V(G)=$ $X, g: X \rightarrow X$ and $T: X \rightarrow C B(X)$. Then $T$ is said to be $g$-graph-preserving if for any $x, y \in X$ such that

$$
(g(x), g(y)) \in E(G) \quad \Rightarrow \quad(u, v) \in E(G) \quad \text { for all } u \in T x \text { and } v \in T y .
$$

Example 2.10 Let $G=(\mathbb{N}, E(G))$ and $E(G)=\{(2 n-1,2 n+1): n \in \mathbb{N}\} \cup\{(2 n, 2 n+2): n>1\}$ $\cup\{(2 n, 2 n+4): n>1\} \cup\{(2 n, 2 n): n>1\} \cup\{(1,1) \cup(6,4)\}$. Let $T: \mathbb{N} \rightarrow C B(\mathbb{N})$ be defined as in Example 2.7 and let $g: \mathbb{N} \rightarrow \mathbb{N}$ be defined by

$$
g(x)= \begin{cases}2 k & \text { if } x=2 k+2, k \in \mathbb{N}, \\ 2 k-1 & \text { if } x=2 k+1, k \in \mathbb{N}, \\ 2 & \text { if } x=1,2 .\end{cases}
$$

We will show that $T$ is $g$-graph-preserving. Let $(g(x), g(y)) \in E(G)$.

If $(g(x), g(y))=(2 k-1,2 k+1)$ for $k \in \mathbb{N}$, then $(x, y)=(2 k+1,2 k+3)$ and $T x=\{2 k+2,2 k+$ $4\}, T y=\{2 k+4,2 k+6\}$ and $(2 k+2,2 k+4) \in E(G),(2 k+2,2 k+6) \in E(G),(2 k+4,2 k+4) \in$ $E(G),(2 k+4,2 k+6) \in E(G)$.

If $(g(x), g(y))=(2 k, 2 k+2)$ or $(2 k, 2 k+4)$ or $(2 k, 2 k)$, then $T x=T y=\{1\}$ and $(1,1) \in E(G)$.

If $(g(x), g(y))=(1,1)$, then $(x, y)=(3,3)$ and $T 3=\{4,6\}$ and $(4,4) \in E(G),(4,6) \in E(G)$, $(6,4) \in E(G)$ and $(6,6) \in E(G)$.

If $(g(x), g(y))=(6,4)$, then $(x, y)=(8,6)$ and $T 8=T 6=\{1\}$ and $(1,1) \in E(G)$. Hence $T$ is $g$-graph-preserving.

\section{Main results}

We start with defining a new type of multi-valued mappings.

Definition 3.1 Let $(X, d)$ be a metric space, $G=(V(G), E(G))$ be a directed graph such that $V(G)=X, g: X \rightarrow X$ and $T: X \rightarrow C B(X) . T$ is said to be a multi-valued weak $G$-contraction with respect to $g$ or $(g, \alpha, L)-G$-contraction if there exists a function $\alpha$ : $[0, \infty) \rightarrow[0,1)$ satisfying $\lim \sup _{r \rightarrow t^{+}} \alpha(r)<1$ for every $t \in[0, \infty)$ and $L \geq 0$ with

$$
H(T x, T y) \leq \alpha(d(g(x), g(y))) d(g(x), g(y))+L D(g(y), T x)
$$

for all $x, y \in X$ such that $(g(x), g(y)) \in E(G)$.

Remark 3.2 If $G=(V(G), E(G))$, where $E(G)=X \times X$ and $g(x)=x, \forall x \in X$, then a $(g, \alpha, L)$ $G$-contraction is a generalized multi-valued $(\alpha, L)$-weak contraction.

Property A ([14] ) For any sequence $\left(x_{n}\right)_{n \in \mathbb{N}}$ in $X$, if $x_{n} \rightarrow x$ and $\left(x_{n}, x_{n+1}\right) \in E(G)$ for $n \in \mathbb{N}$, then there is a subsequence $\left(x_{k_{n}}\right)_{n \in \mathbb{N}}$ with $\left(x_{k_{n}}, x\right) \in E(G)$ for $n \in \mathbb{N}$. 
Theorem 3.3 Let $(X, d)$ be a complete metric space and $G=(V(G), E(G))$ be a directed graph such that $V(G)=X$, and let $g: X \rightarrow X$ be a surjective mapping. If $T: X \rightarrow C B(X)$ is a multi-valued mapping satisfying the following properties:

(1) $T$ is a g-graph-preserving mapping;

(2) there exists $x_{0} \in X$ such that $\left(g\left(x_{0}\right), y\right) \in E(G)$ for some $y \in T x_{0}$;

(3) $X$ has Property A;

(4) $T$ is a $(g, \alpha, L)$-G-contraction;

then there exists $u \in X$ such that $g(u) \in T u$.

Proof Since $g$ is surjective, there exists $x_{1} \in X$ such that $g\left(x_{1}\right) \in T x_{0}$. By (2) we obtain $\left(g\left(x_{0}\right), g\left(x_{1}\right)\right) \in E(G)$. We can choose $n_{1} \in \mathbb{N}$ such that

$$
\left[\alpha\left(d\left(g\left(x_{0}\right), g\left(x_{1}\right)\right)\right)\right]^{n_{1}} \leq\left[1-\alpha\left(d\left(g\left(x_{0}\right), g\left(x_{1}\right)\right)\right)\right] d\left(g\left(x_{0}\right), g\left(x_{1}\right)\right) .
$$

By Lemma 2.1, there exists $g\left(x_{2}\right) \in T x_{1}$ such that

$$
d\left(g\left(x_{1}\right), g\left(x_{2}\right)\right) \leq H\left(T x_{0}, T x_{1}\right)+\left[\alpha\left(d\left(g\left(x_{0}\right), g\left(x_{1}\right)\right)\right)\right]^{n_{1}} .
$$

Since $\left(g\left(x_{0}\right), g\left(x_{1}\right)\right) \in E(G), g\left(x_{1}\right) \in T x_{0}, g\left(x_{2}\right) \in T x_{1}$ and $T$ is a $g$-graph-preserving mapping, we have $\left(g\left(x_{1}\right), g\left(x_{2}\right)\right) \in E(G)$. Moreover, by (3.1) and (3.2), we get

$$
\begin{aligned}
d\left(g\left(x_{1}\right), g\left(x_{2}\right)\right) \leq & H\left(T x_{0}, T x_{1}\right)+\left[\alpha\left(d\left(g\left(x_{0}\right), g\left(x_{1}\right)\right)\right)\right]^{n_{1}} \\
\leq & \alpha\left(d\left(g\left(x_{0}\right), g\left(x_{1}\right)\right)\right)\left(d\left(g\left(x_{0}\right), g\left(x_{1}\right)\right)\right)+L D\left(g\left(x_{1}\right), T x_{0}\right) \\
& +\left[\alpha\left(d\left(g\left(x_{0}\right), g\left(x_{1}\right)\right)\right)\right]^{n_{1}} \\
\leq & \alpha\left(d\left(g\left(x_{0}\right), g\left(x_{1}\right)\right)\right)\left(d\left(g\left(x_{0}\right), g\left(x_{1}\right)\right)\right)+\left[\alpha\left(d\left(g\left(x_{0}\right), g\left(x_{1}\right)\right)\right)\right]^{n_{1}} \\
\leq & d\left(g\left(x_{0}\right), g\left(x_{1}\right)\right) .
\end{aligned}
$$

Next, we can choose $n_{2}>n_{1}$ such that

$$
\left[\alpha\left(d\left(g\left(x_{1}\right), g\left(x_{2}\right)\right)\right)\right]^{n_{2}} \leq\left[1-\alpha\left(d\left(g\left(x_{1}\right), g\left(x_{2}\right)\right)\right)\right] d\left(g\left(x_{1}\right), g\left(x_{2}\right)\right) .
$$

By Lemma 2.1, there exists $g\left(x_{3}\right) \in T x_{2}$ such that

$$
d\left(g\left(x_{2}\right), g\left(x_{3}\right)\right) \leq H\left(T x_{1}, T x_{2}\right)+\left[\alpha\left(d\left(g\left(x_{1}\right), g\left(x_{2}\right)\right)\right)\right]^{n_{2}} .
$$

By the above two inequalities and $\left(g\left(x_{1}\right), g\left(x_{2}\right)\right) \in E(G)$, we get

$$
\begin{aligned}
d\left(g\left(x_{2}\right), g\left(x_{3}\right)\right) \leq & H\left(T x_{1}, T x_{2}\right)+\left[\alpha\left(d\left(g\left(x_{1}\right), g\left(x_{2}\right)\right)\right)\right]^{n_{2}} \\
\leq & \alpha\left(d\left(g\left(x_{1}\right), g\left(x_{2}\right)\right)\right)\left(d\left(g\left(x_{1}\right), g\left(x_{2}\right)\right)\right)+L D\left(g\left(x_{2}\right), T x_{1}\right) \\
& +\left[\alpha\left(d\left(g\left(x_{1}\right), g\left(x_{2}\right)\right)\right)\right]^{n_{2}} \\
\leq & \alpha\left(d\left(g\left(x_{1}\right), g\left(x_{2}\right)\right)\right)\left(d\left(g\left(x_{1}\right), g\left(x_{2}\right)\right)\right)+\left[\alpha\left(d\left(g\left(x_{1}\right), g\left(x_{2}\right)\right)\right)\right]^{n_{2}} \\
\leq & d\left(g\left(x_{1}\right), g\left(x_{2}\right)\right) .
\end{aligned}
$$


By induction, we obtain a sequence $\left\{g\left(x_{k}\right)\right\}$ in $X$ and a sequence $\left\{n_{k}\right\}$ of positive integers with the property that for each $k \in \mathbb{N}, g\left(x_{k+1}\right) \in T x_{k},\left(g\left(x_{k}\right), g\left(x_{k+1}\right)\right) \in E(G)$ and

$$
\left[\alpha\left(d\left(g\left(x_{k-1}\right), g\left(x_{k}\right)\right)\right)\right]^{n_{k}} \leq\left[1-\alpha\left(d\left(g\left(x_{k-1}\right), g\left(x_{k}\right)\right)\right)\right] d\left(g\left(x_{k-1}\right), g\left(x_{k}\right)\right),
$$

and

$$
d\left(g\left(x_{k}\right), g\left(x_{k+1}\right)\right) \leq H\left(T x_{k-1}, T x_{k}\right)+\left[\alpha\left(d\left(g\left(x_{k-1}\right), g\left(x_{k}\right)\right)\right)\right]^{n_{k}} .
$$

Therefore $d\left(g\left(x_{k}\right), g\left(x_{k+1}\right)\right) \leq d\left(g\left(x_{k-1}\right), g\left(x_{k}\right)\right)$ for any $k \in \mathbb{N}$, i.e., $\left\{g\left(x_{k}\right)\right\}$ is a non-increasing sequence. Thus it follows from Lemma 2.2 that $\left\{g\left(x_{k}\right)\right\}$ is a Cauchy sequence in $X$. Since $X$ is complete, there exists $u \in X$ such that $\lim _{k \rightarrow \infty} g\left(x_{k}\right)=g(u)$. By assumption (3), we have a subsequence $g\left(x_{k_{n}}\right)$ such that $\left(g\left(x_{k_{n}}\right), g(u)\right) \in E(G)$ for any $n \in \mathbb{N}$. Thus we get

$$
\begin{aligned}
D(g(u), T u) & \leq d\left(g(u), g\left(x_{k_{n}+1}\right)\right)+D\left(g\left(x_{k_{n}+1}\right), T u\right) \\
& \leq d\left(g(u), g\left(x_{k_{n}+1}\right)\right)+H\left(T x_{k_{n}}, T u\right) \\
& \leq d\left(g(u), g\left(x_{k_{n}+1}\right)\right)+\alpha\left(d\left(g\left(x_{k_{n}}\right), g(u)\right)\right) d\left(g\left(x_{k_{n}}\right), g(u)\right)+L D\left(g(u), T x_{k_{n}}\right) \\
& \leq d\left(g(u), g\left(x_{k_{n}+1}\right)\right)+\alpha\left(d\left(g\left(x_{k_{n}}\right), g(u)\right)\right) d\left(g\left(x_{k_{n}}\right), g(u)\right)+L d\left(g(u), g\left(x_{k_{n}-1}\right)\right) .
\end{aligned}
$$

Since $g\left(x_{k_{n}}\right)$ converges to $g(u)$ as $n \rightarrow \infty$, it follows that $D(g(u), T u)=0$. Since Tu is closed, we conclude that $g(u) \in T u$.

Corollary 3.4 Let $(X, d)$ be a metric space endowed with a partial order $\leq, g: X \rightarrow X$ be surjective and $T: X \rightarrow C B(X)$ be a multivalued mapping. Suppose that

(1) $T$ is $g$-increasing;

(2) there exist $x_{0} \in X$ and $u \in T x_{0}$ such that $g\left(x_{0}\right)<u$;

(3) for each sequence $\left\{x_{k}\right\}$ such that $g\left(x_{k}\right)<g\left(x_{k+1}\right)$ for all $k \in \mathbb{N}$ and $g\left(x_{k}\right)$ converges to $g(x)$, for some $x \in X$, then $g\left(x_{k}\right)<g(x)$ for all $k \in \mathbb{N}$;

(4) there exists $\alpha:[0, \infty) \rightarrow[0,1)$ satisfying $\lim \sup _{r \rightarrow t^{+}} \alpha(r)<1$ for every $t \in[0, \infty)$ and $L>0$ such that

$$
H(T x, T y) \leq \alpha(d(g(x), g(y)))(d(g(x), g(y)))+L D(g(y), T x)
$$

for any $x, y \in X$ with $g(x)<g(y)$;

(5) the metric $d$ is complete.

Then there exists $u \in X$ such that $g(u) \in T u$.

Proof Define $G=(V(G), E(G))$ by $V(G)=X$ and $E(G)=\{(x, y): x<y\}$. Let $x, y \in X$ such that $(g(x), g(y)) \in E(G)$. Then $g(x)<g(y)$ so $T x \prec T y$. For any $u \in T x$ and $v \in T y$, we have $u<v$, i.e., $(u, v) \in E(G)$. So $T$ is graph-preserving. By assumption (2), there exist $x_{0}$ and $u \in T x_{0}$ such that $g\left(x_{0}\right)<u$, so $\left(g\left(x_{0}\right), u\right) \in E(G)$. Hence (2) of Theorem 3.3 is satisfied. It is easy to see that (3) and (4) of Theorem 3.3 are also satisfied. Therefore Corollary 3.4 is obtained directly by Theorem 3.3 .

If we put $g(x)=x$ for all $x \in X$ in Corollary 3.4, we obtain the following result. 
Corollary 3.5 Let $(X, d)$ be a metric space endowed with a partial order $\leq$ and $T: X \rightarrow$ $C B(X)$ be a multivalued mapping. Suppose that

(1) $T$ is increasing;

(2) there exists $x_{0} \in X$ such that $x_{0}<T x_{0}$;

(3) for each sequence $\left\{x_{n}\right\}$ such that $x_{n}<x_{n+1}$ for all $n \in \mathbb{N}$ and $x_{n}$ converges to $x$, for some $x \in X$, then $x_{n}<x$ for all $n \in \mathbb{N}$;

(4) there exist $\alpha:[0, \infty) \rightarrow[0,1)$ and $L>0$ such that

$$
H(T x, T y) \leq \alpha(d(x, y))(d(x, y))+L D(y, T x)
$$

for any $x, y \in X$ with $x<y$, where $\lim \sup _{r \rightarrow t^{+}} \alpha(r)<1$ for every $t \in[0, \infty)$;

(5) the metric $d$ is complete.

Then there exists $u \in X$ such that $u \in T u$.

Remark 3.6 Theorem 4 in [3] is directly obtained from Theorem 3.3 by setting $G=$ $(V(G), E(G))$, where $V(G)=X, E(G)=X \times X$ and $g(x)=x$ for all $x \in X$.

Example 3.7 Let $X=\{0,1,3,4,6,7,9,10,11\}, d(x, y)=|x-y|, x, y \in E(G), E(G)=\{(1,4)$, $(1,7),,(4,4)(4,7),(7,4),(7,7)\} \cup\{(0,3),(0,6),(3,3),(3,6),(6,3),(6,6)\} \cup\{(9,10),(10,9)$, $(10,10),(10,11),(11,10),(11,11)\}$ and $T: X \rightarrow C B(X)$ be defined by

$$
T(x)= \begin{cases}\{1,4\} & \text { if } x=0 \\ \{0,3\} & \text { if } x=1 \\ \{4,7\} & \text { if } x=3,6 \\ \{3,6\} & \text { if } x=4,7 \\ \{10,11\} & \text { if } x=9,10,11\end{cases}
$$

We note that

$$
H(T(1), T(4))=3 \not \leq k d(1,4) \quad \text { for each } k \in[0,1) .
$$

This means that $T$ does not satisfy Nadler's theorem. We will show that $T$ is a weak contraction with $\alpha(x)=\frac{1}{2}$ and $L=2$.

Let $(x, y) \in E(G)$.

If $(x, y) \in\{(3,3),(3,6),(6,3),(6,6),(4,4),(4,7),(7,4),(7,7),(9,10),(10,9),(10,10),(10,11)$, $(11,10),(11,11)\} \in E(G)$, we have

$$
H(T x, T y)=0 \leq \frac{1}{2} d(x, y)+2 d(y, T x)
$$

If $(x, y)=(1,4)$, we have

$$
H(T 1, T 4)=3 \leq \frac{1}{2} d(1,4)+2 d(4, T 1) .
$$

If $(x, y)=(1,7)$, we have

$$
H(T 1, T 7)=3 \leq \frac{1}{2} d(1,7)+2 d(7, T 1) .
$$


If $(x, y)=(0,3)$, we have

$$
H(T 0, T 3)=3 \leq \frac{1}{2} d(0,3)+2 d(3, T 0) .
$$

If $(x, y)=(0,6)$, we have

$$
H(T 0, T 6)=3 \leq \frac{1}{2} d(0,6)+2 d(6, T 0) .
$$

Hence $T$ is an $(\alpha, g, L)$-G-contraction. To show that $T$ is graph-preserving, let $(x, y) \in$ $E(G)$. If $(x, y)=(1,4)$ or $(x, y)=(1,7)$, then $T x=\{0,3\}, T y=\{3,6\}$, and we see that $(0,3),(0,6),(3,3),(3,6) \in E(G)$. If $(x, y) \in\{(4,4),(4,7),(7,4)\}$, then $T x=\{3,6\}=T y$ and $(3,3),(3,6),(6,3),(6,6) \in E(G)$. If $(x, y)=(0,3)$ or $(x, y)=(0,6)$, then $T x=\{1,4\}, T y=\{4,7\}$ and we see that $(1,4),(1,7),(4,4),(4,7) \in E(G)$. If $(x, y) \in\{(3,3),(3,6),(6,3),(6,6)\}$, then $T x=\{4,7\}=T y$ and we see that $(4,4),(4,7),(7,4),(7,7) \in E(G)$. If $(x, y) \in\{(9,10),(10,9)$, $(10,11),(11,10)\}$, then $T x=\{10,11\}=T y$ and we see that $(10,10),(10,11),(11,10),(11,11) \in$ $E(G)$. Hence $T$ is graph-preserving. By the definition of $T$ and $G$, we see that $10 \in T 9=$ $\{10,11\}$ and $(9,10) \in E(G)$, that is, condition (2) of Theorem 3.3 is satisfied. It is easy to see that $X$ has Property A. Therefore all the conditions of Theorem 3.3 are satisfied, so $T$ has a fixed point and we see that $\operatorname{Fix}(T)=\{10,11\}$.

Next, we give an example of a map which lacks assumption (2) and has no fixed point.

Example 3.8 Let $X:=\{0,1,3,4,6,7,9,10,11\}, d(x, y)=|x-y|, x, y \in X, E(G):=\{(1,4)$, $(4,7)\} \cup\{(0,3),(3,0),(3,6),(6,3),(0,6),(6,0)\} \cup\{(9,10),(10,9),(10,11)\}$ and $T: X \rightarrow C B(X)$ be defined by

$$
T(x)= \begin{cases}\{1,4\} & \text { if } x=0, \\ \{0,3\} & \text { if } x=1, \\ \{4,7\} & \text { if } x=3,6, \\ \{3,6\} & \text { if } x=4,7, \\ \{0\} & \text { if } x=9,10,11\end{cases}
$$

and let $g: X \rightarrow X$ be an identity map. The same as in Example 3.7, $T$ is a graph-preserving mapping and $T$ is a $\left(g, \frac{1}{2}, 2\right)$ - $G$-contraction. Moreover, we can easily check that condition (2) of Theorem 3.3 does not hold and we note that $T$ has no fixed point.

\section{Competing interests}

The authors declare that they have no competing interests.

Authors' contributions

All authors contributed equally to the writing of this paper. All authors read and approved the final manuscript.

\section{Acknowledgements}

The authors would like to thank the referees for their valuable comments and suggestions. The first author would like to thank Science Achievement Scholarship of Thailand (SAST). This paper was supported by Chiang Mai University, Chiang Mai, Thailand. 
References

1. Nadler, S: Multi-valued contraction mappings. Pac. J. Math. 20(2), 475-488 (1969)

2. Alghamdi, MA, Berinde, V, Shahzad, N: Fixed points of multivalued nonself almost contractions. J. Appl. Math. 2013, Article ID 621614 (2013)

3. Berinde, M, Berinde, V: On a general class of multi-valued weakly Picard mappings. J. Math. Anal. Appl. 326, 772-782 (2007)

4. Berinde, V, Pacurar, M: The role of Pompeiu-Hausdorff metric in fixed point theory. Creative Math. Inform. 22(2), 143-150 (2013)

5. Beg, I, Butt, AR: The contraction principle for set valued mappings on a metric space with graph. Comput. Math. Appl. 60, 1214-1219(2010)

6. Dinevari, T, Frigon, M: Fixed point results for multivalued contractions on a metric space with a graph. J. Math. Anal. Appl. 405, 507-517 (2013)

7. Reich, S: Fixed points of contractive functions. Boll. Unione Mat. Ital. 5, 26-42 (1972)

8. Mizoguchi, N, Takahashi, W: Fixed point theorems for multivalued mappings on complete metric spaces. J. Math. Anal. Appl. 141, 177-188 (1989)

9. Ran, ACM, Reurings, MCB: A fixed point theorem in partially ordered sets and some applications to matrix equations. Proc. Am. Math. Soc. 132(5), 1435-1443 (2003)

10. Beg, I, Butt, AR: Common fixed point for generalized set valued contractions satisfying an implicit relation in partially ordered metric spaces. Math. Commun. 15,65-75 (2010)

11. Beg, I, Latif, A: Common fixed point and coincidence point of generalized contractions in ordered metric spaces. Fixed Point Theory Appl. (2012). doi:10.1186/1687-1812-2012-229

12. Beg, I, Butt, AR: Fixed point for set valued mappings satisfying an implicit relation in partially ordered metric spaces. Nonlinear Anal. 71, 3699-3704 (2009)

13. Bhaskar, TG, Laskhmikantham, V: Fixed point theorems in partially ordered metric spaces and applications. Nonlinear Anal. TMA 65(7), 1379-1393 (2006)

14. Jachymski, J: The contraction principle for mappings on a metric with a graph. Proc. Am. Math. Soc. 1(136), 1359-1373 (2008)

10.1186/1687-1812-2014-70

Cite this article as: Tiammee and Suantai: Coincidence point theorems for graph-preserving multi-valued mappings. Fixed Point Theory and Applications 2014, 2014:70

\section{Submit your manuscript to a SpringerOpen ${ }^{\circ}$ journal and benefit from:}

- Convenient online submission

- Rigorous peer review

- Immediate publication on acceptance

- Open access: articles freely available online

- High visibility within the field

- Retaining the copyright to your article 